\title{
Adjunct extended-release valproate semisodium in late life schizophrenia
}

\author{
Martha Sajatovic $^{1 *}$, Nicoleta Coconcea ${ }^{1,2}$, Rosalinda V. Ignacio ${ }^{3,4}$, Frederic C. Blow ${ }^{3,4}$, \\ Robert W. Hays ${ }^{2}$, Kristin A. Cassidy ${ }^{1}$ and William J. Meyer ${ }^{1}$ \\ ${ }^{1}$ Department of Psychiatry, Case Western Reserve University, Cleveland, Ohio, USA \\ ${ }^{2}$ NorthEast Ohio Health Services, Cleveland, Ohio, USA \\ ${ }^{3}$ Serious Mental Illness Treatment Research and Evaluation Center (SMITREC), Health Services Research and \\ Development, Ann Arbor VA Healthcare System, USA \\ ${ }^{4}$ Department of Psychiatry, University of Michigan, Ann Arbor, Michigan, USA
}

\section{SUMMARY}

Objective Adjunctive anticonvulsant medications may benefit some individuals with schizophrenia, however data on adjunct anticonvulsants in older adults with schizophrenia is limited. This prospective, 12-week open label study evaluated adjunct extended-release valproate semisodium (divalproex) in 20 older adults with schizophrenia.

Methods The study was conducted at an academic psychiatry clinic in the mid-western United States. Participants were self-referred from posted advertisements or referred by clinic practitioners. Extended-release valproate semisodium was added onto antipsychotic treatment. Individuals with active substance use disorders or active significant medical comorbidity were excluded. Primary outcome measures included the Positive and Negative Syndrome Scale (PANSS), Geriatric Depression Scale (GDS) and Global Assessment Scale (GAS). Tolerability was evaluated via patient self-reported side effects, change from baseline in body weight and change on abnormal movement scales.

Results Patients (mean age 61 years, range 49.8-79.2 years) had significant reductions in psychosis scores as measured by the Positive and Negative Syndrome Scale (PANSS) $p<0.01$, as well as in global functioning as measured by the Global Assessment Scale (GAS) $p<0.01$ and depression as measured by the Geriatric Depression Scale (GDS) $p<0.05$. Mean dose of extended-release valproate semisodium was $587.50 \mathrm{mg} / \mathrm{day} \mathrm{SD} \pm 247.02$. Extended-release valproate semisodium was well tolerated in this older adult population. The primary adverse effect was sedation, which appeared to be relatively dose and titration-speed dependent. Weight change was not significant.

Conclusion While extended-release valproate semisodium appears efficacious and well tolerated in older adults with schizophrenia, data from larger, controlled trials is needed. Copyright (C) 2007 John Wiley \& Sons, Ltd.

KEY WORDS - schizophrenia; valproate semisodium; elderly; anticonvulsants

\section{INTRODUCTION}

In clinical settings anticonvulsants are sometimes utilized to augment treatment among individuals with schizophrenia (Citrome et al., 2000), and there are reports suggesting that augmentation anticonvulsant

*Correspondence to: Dr M. Sajatovic, Department of Psychiatry, University Hospitals of Cleveland, 11100 Euclid Avenue, Cleveland, OH 44106, USA. E-mail: martha.sajatovic@uhhs.com therapy may improve schizophrenia symptoms (Wassef et al., 2000; Casey et al., 2003; Citrome et al., 2004).

The issue of older adults with schizophrenia has gained attention due to the growing proportion of elderly world-wide (CDC, 2004; Jeste et al., 1999). In geropsychiatric settings up to $65 \%$ of older adults with schizophrenia/schizoaffective disorder receive anticonvulsant medication (Sajatovic et al., 2004). Although utilization of anticonvulsants, valproate semisodium in particular, is routinely seen in clinical settings, how this augmentation specifically benefits older adults 
with schizophrenia is not clear. This is a first report of a prospective trial of adjunctive extended-release valproate semisodium (divalproex) therapy focused on older adults with schizophrenia. We hypothesized that adjunctive extended-release valproate semisodium would be well-tolerated and associated with improvements in symptoms, level of functioning, and general health status.

\section{METHODS}

This was extended-release open-label, 12-week prospective trial of add-on valproate semisodium therapy in 20 older adults with schizophrenia as confirmed by the Mini International Neuropsychiatric Interview (MINI; Sheehan et al., 1998). The study was conducted at an academic psychiatry clinic in the mid-western United States. Participants were recruited in response to self- referrals from posted advertisements and by referrals from mental health practitioners at the academic clinic and a nearby community mental health clinic. Individuals were considered sub-optimally responsive to current antipsychotic medications if, based upon either self-report or report of care providers, they had remaining symptoms of schizophrenia that either affected their ability to meet daily needs or their ability to interact with others. All patients provided written informed consent, and the study was approved by the local institutional review board (IRB). Eligible subjects were receiving antipsychotic medications for the treatment of schizophrenia. Individuals with acute medical illness, including those positive for Hepatitis $\mathrm{C}$, and those with active substance abuse were excluded. Individuals were only considered for participation if they had not received a valproate trial in the past.

Enrolled individuals received adjunctive, openlabel valproate semisodium, initially started as valproate semisodium delayed-release $250 \mathrm{mg}$ at bedtime for two weeks, then changed to valproate semisodium extended-release $500 \mathrm{mg}$ at bedtime. Medication was administered on an outpatient/ambulatory basis, and adjusted as tolerated to target serum levels of 50 $100 \mu \mathrm{g} / \mathrm{mL}$. In cases where sedation or other side effects occurred, dosage was reduced. Valproate semisodium was prescribed in a single dose at bedtime.

The primary outcome measure was change from baseline on a schizophrenia psychopathology assessment, the Positive and Negative Syndrome Scale (PANSS; Kay et al., 1987). Secondary outcomes included change from baseline on a measure of depression [the Geriatric Depression Scale (GDS: Yesavage et al., 1982)], overall change in functioning [Global Assessment Scale (GAS; Guy, 1976)] and change in general health status [Short Form 36 Health Survey (SF-36; Ware and Sherbourne, 1992)] and cognitive status [Mini-Mental State Examination (MMSE; Folstein et al., 1975)]. Extrapyramidal symptoms were evaluated with the Abnormal Involuntary Movement Scale (AIMS; Guy, 1976), the Barnes Akathisia Scale (BAS; Barnes, 1989), and the Simpson Angus Neurological Rating Scale (SAS; Simpson and Angus, 1970).

All rating scale measures were conducted at baseline, and at weeks 2,4,8 and 12 (end of study). All patients had baseline assessment of basic serum chemistry, metabolic profile, complete blood count (CBC) with differential, vital signs, weight and electrocardiogram. Vital signs and weight were assessed at each study visit, and laboratory testing was repeated again at end of study. Serum total valproate levels were obtained by week 4 , and at weeks 8 and 12 .

Efficacy and safety results were calculated using descriptive statistics. Scores for each rating scale over time were evaluated using paired $t$-tests comparing baseline and last available assessment of each measure. The intent-to-treat (ITT) population was defined as individuals who received at least one dose of valproate semisodium.

\section{RESULTS \\ Clinical characteristics of the sample}

Table 1 illustrates sample baseline clinical characteristics. Mean age was 61.5 years, $\mathrm{SD} \pm 9.4$, range $49.8-79.2$ years. This was a chronically mentally ill population, with mean age of $31.2 \pm$ SD 18.1 years at illness onset, and mean duration of illness of $29.2 \pm 13.3$ years. The majority of patients had moderate baseline symptoms of schizophrenia with a mean PANSS score of $72.65 \pm 17.17$, mean PANSS negative sub-scale $18.20 \pm 6.30$, and mean PANSS positive subscale $17.90 \pm 3.51$. This was a non-demented population with a mean MMSE score of $27.50 \pm 1.73$, range 24-30.

Concomitant antipsychotic medications included risperidone $(n=6,33 \%)$, olanzapine $(n=4,20 \%)$, aripiprazole $(n=4,20 \%)$, quetiapine $(n=3,15 \%)$, and clozapine $(n=1,5 \%)$. One individual was on both olanzapine and long-acting injectable risperidone, and one individual was on both olanzapine and aripiprazole. There were four individuals on conventional 
Table 1. Clinical characteristics of 20 older adults with schizophrenia who received adjunctive (extended-release) valproate semisodium therapy

\begin{tabular}{|c|c|c|}
\hline Variable & Value N (\%) & Baseline to LOCF $t$-test $p$-value \\
\hline \multicolumn{3}{|l|}{ Sex } \\
\hline Male & $4(20)$ & \multirow[t]{2}{*}{$\mathrm{n} / \mathrm{a}$} \\
\hline Female & $16(80)$ & \\
\hline \multicolumn{3}{|l|}{ Age, years } \\
\hline Mean \pm SD & $61.1 \pm 9.6$ & \multirow[t]{2}{*}{$\mathrm{n} / \mathrm{a}$} \\
\hline Range & $49.8-79.2$ & \\
\hline \multicolumn{3}{|l|}{ Ethnicity } \\
\hline Caucasian & $9(45)$ & \multirow[t]{2}{*}{$\mathrm{n} / \mathrm{a}$} \\
\hline African-American & $11(55)$ & \\
\hline \multicolumn{3}{|c|}{ Antipsychotic medication treatment* } \\
\hline Risperidone & $6(33)$ & \multirow{6}{*}{$\mathrm{n} / \mathrm{a}$} \\
\hline Olanzapine & $4(20)$ & \\
\hline Typical compounds & $4(20)$ & \\
\hline Aripiprazole & $4(20)$ & \\
\hline Quetiapine & $3(15)$ & \\
\hline Clozapine & $1(5)$ & \\
\hline \multicolumn{3}{|c|}{ Most common comorbid medical conditions } \\
\hline Hypertension & $9(45)$ & \multirow{7}{*}{$\mathrm{n} / \mathrm{a}$} \\
\hline Diabetes & $8(40)$ & \\
\hline Hyperlipidemia & $6(30)$ & \\
\hline Coronary Artery Disease & $5(20)$ & \\
\hline Chronic Renal Insufficiency & $3(15)$ & \\
\hline Morbid Obesity & $3(15)$ & \\
\hline Arthritis & $3(15)$ & \\
\hline \multicolumn{3}{|l|}{ Baseline PANSS } \\
\hline Mean \pm SD & $72.65 \pm 17.17$ & $<0.001$ \\
\hline \multicolumn{3}{|l|}{ Baseline GDS } \\
\hline Mean \pm SD & $15.72 \pm 4.56$ & 0.017 \\
\hline \multicolumn{3}{|l|}{ Baseline GAS } \\
\hline Mean \pm SD & $46.9 \pm 14.34$ & $<0.001$ \\
\hline \multicolumn{3}{|l|}{ Baseline MMSE } \\
\hline Mean \pm SD & $27.5 \pm 1.73$ & 0.585 \\
\hline \multicolumn{3}{|l|}{ Baseline SF-36 } \\
\hline Mean $\mathrm{PCS} \pm \mathrm{SD}$ & $35.2 \pm 10.05$ & 0.569 \\
\hline Mean $\mathrm{MCS} \pm \mathrm{SD}$ & $37.1 \pm 13.74$ & 0.027 \\
\hline \multicolumn{3}{|c|}{ Dose of valproate semisodium $\mathrm{mg} /$ day at end of study } \\
\hline Mean \pm SD & $587.5 \pm 247.02$ & $\mathrm{n} / \mathrm{a}$ \\
\hline Range & $250-1000$ & \\
\hline
\end{tabular}

GAS $=$ Global Assessment of Functioning; GDS $=$ Geriatric Depression Scale; MMSE $=$ Mini Mental State Examination; PANSS $=$ Positive and Negative Syndrome Scale; SF-36 = 36-item Short Form Health Survey; PCS = Physical Composite; MCS = Mental Composite. *Some individuals on more than one antipsychotic compound.

antipsychotics (haloperidol $n=3$, thiothixene $n=1$ ), including two on long-acting injectable haloperidol. During the study there were five individuals who had reductions in their antipsychotic medication dosing (four individuals to reduce extrapyramidal symptoms (haloperidol $n=2$, thiothixene $n=1$, risperidone $n=1$ ), and one individual on olanzapine to attempt to reduce propensity for weight gain). Baseline chlorpromazine (CPZ) equivalents (Fuller and Sajatovic, 2007) for individuals that were on oral antipsychotic medications was $375 \mathrm{CPZ}$ equivalents/day ( $\mathrm{SD} \pm 268.3$, range 100-1,250 CPZ equivalents/day), while endpoint $\mathrm{CPZ}$ equivalents was $303 \mathrm{CPZ}$ equivalents/day ( $\mathrm{SD} \pm 288.5)$, a difference that was not statistically significant $(p>0.05)$.

Comorbid medical conditions were common, and included hypertension $(n=9,45 \%)$, diabetes $(n=8$, $40 \%)$, hyperlipidemia/cholesterolemia $(n=6,30 \%)$, coronary artery disease $(n=5,20 \%)$, chronic renal insufficiency $(n=3,15 \%)$, arthritis $(n=3,15 \%)$, morbid obesity $(n=3,15 \%)$ and one individual each (5\%) with chronic pain, chronic osteomyelitis, GERD, Parkinson's disease, hypothyroidism, sarcoidosis and seizure disorder. 


\section{Efficacy}

Significant improvements were seen in PANSS $(p<0.001)$, GAS $(p<0.001)$ and GDS ratings $(p<0.02)$. The MMSE scores did not change significantly $(p=0.585)$. There was no significant difference in the Physical Composite Score (PCS) subscale of the SF-36 $(p=0.569)$, but there was a significant improvement in the Mental Composite Score (MCS) subscale $(p<0.05)$.

\section{Tolerability:}

Extended-release valproate semisodium was fairly well tolerated in this population. Five patients prematurely discontinued study medication due to nonadherence with study medication in 4 individuals and adverse medical event unrelated to study medication (groin abscess) in one individual. Adverse effects included sedation in six individuals (30\%), and more rarely constipation, dry mouth, orthostasis (one individual, $5 \%$ for each). Mean body weight was $89.4 \pm$ $19.8 \mathrm{~kg}$ at baseline and $91.6 \pm 21.4 \mathrm{~kg}$ at endpoint $(p=0.21)$. EPS ratings showed significant improvement in AIMS scores $(p<0.05)$ and BAS scores $(p<0.05)$, but no significant change in SAS scores $(p=0.20)$.

Mean daily dose of extended-release valproate semisodium was $587.5 \pm 247.02 \mathrm{mg} /$ day, range 250 $1000 \mathrm{mg} /$ day. Mean serum level at study end-point was $40.86 \pm 25.29 \mu \mathrm{g} / \mathrm{mL}$. There were no significant changes on laboratory testing or in vital signs.

\section{DISCUSSION}

This open-label, pilot study suggests that adjunctive extended-release valproate semisodium is relatively well tolerated and may be associated with improvements in psychopathology among older adults with schizophrenia. While $25 \%$ of individuals in the study presented here dropped out prematurely, none did so because of adverse events related to study medication. Recent large-scale treatment trials involving mixed age patients with schizophrenia (Lieberman et al., 2005; Jones et al., 2006) demonstrate early medication discontinuation or medication switching rates in the order of $46-74 \%$. Low persistence with medication treatment appears to be a pervasive problem among populations with schizophrenia that complicates interpretation of studies of schizophrenia treatments.

Casey and colleagues (2003) conducted a double blind, randomized study of valproate semisodium with an antipsychotic agent for mixed age individuals with schizophrenia. Patients were treated with either olanzapine or risperidone monotherapy, valproate semisodium plus olanzapine or valproate semisodium plus risperidone for 28 days. Improvements from baseline were observed in all treatment groups, with statistically significant treatment differences favoring combination treatments on the Positive and Negative Syndrome Scale (PANSS) total score by day 3 and persisting to day $21(p<0.05)$, but not to day 28 $(p=0.108)$. An 84-day study failed to replicate the apparent early robust response to adjunct valproate (http://www.clinicalstudyresults.org/documents/companystudy_782_0.pdf.) Similarly, a Cochrane Database review (Basan et al., 2004) that examined the effectiveness of valproate as an adjunct to antipsychotic medications in mixed age schizophrenia populations noted no significant effect of using valproate as an adjunct to antipsychotic therapy. Randomized, controlled trials with valproate semisodium have also been conducted to evaluate effects on behavioral symptoms in elderly dementia populations (Porsteinsson et al., 2001; Sival et al., 2002; Tariot et al., 2005). However, the findings are consistent with the relatively unpromising results across varying psychotropic compounds for symptoms of dementia (Sink et al., 2005).

In spite of the inconsistent/negative randomized, controlled trials (RCTs) in general schizophrenia populations, it has been suggested that valproate may remain a useful adjunct in sub-populations with schizophrenia who are sub-optimally responsive to antipsychotic monotherapy or who have other atypical presentations such as prominent mood or anxiety symptoms or those with aggressive behavior (Basan et al., 2004; Citrome et al., 2004; Stahl, 2004; Townsend and Wilson, 2005; Gobbi et al., 2006). Elderly individuals, who may be unusually sensitive to adverse effects of antipsychotics, or those older adults who have remaining symptoms even with antipsychotic medication treatments may potentially benefit from adjunctive valproate.

Individuals in the study reported here received relatively modest doses of valproate semisodium (mean dose $587.5 \mathrm{mg} /$ day), considerably lower than the doses reported in the study by Casey and colleagues (2003). In the mixed age trial conducted by Casey and colleagues (2003), 29\% of individuals experienced somnolence, which was the most common reported side effect of all treatments. Consistent with the report by Basan and colleagues (2004), sedation was the most common adverse effect (30\% of individuals) among older adults in this study who received adjunct valproate semisodium. In some 
instances, dosing increases in our older adults sample were limited by subjective experience of sedation or tiredness.

The mechanism of action by which adjunct anticonvulsant medications may be helpful in refractory or resistant psychosis is unclear. It has been suggested that diminished prefrontal cortical dopamine activity may contribute to impaired cognition among individuals with schizophrenia, and that use of adjunct anticonvulsant therapy may enhance release of cortical dopamine. Ichikawa and colleagues (2005) recently reported that valproate, when combined with antipsychotics, produces greater increases in prefrontal cortical dopamine release than either type of drug alone via a mechanism dependent upon 5HT (1A) receptor activation. Alternatively, Wassef and colleagues $(1999,2003)$ have suggested that abnormalities in the gamma-aminobutyric acid (GABA) neurotransmitter system implicated in the pathogenesis of schizophrenia, may be at least partially corrected by GABA-ergic agonists such as valproate.

While the MMSE did not demonstrate change with valproate semisodium therapy in this non-demented population, it is possible that more subtle improvements in cognition could have been at least in part demonstrated by changes seen on the other psychopathology ratings such as the PANSS. It is known that valproate co-administration can increase plasma concentrations and area under the plasma concentration-time curve (AUC) of the anticonvulsants lamotrigine and carbamazepine, lorazepam and some antidepressants (Calvo et al., 1986; Anderson, 1998; Fuller, 2007). Adjunctive valproate therapy has been reported to have minimal effects on steady state serum levels of some antipsychotic compounds (Citrome et al., 2005; Fuller, 2007).

In this study, older adults were initially begun on valproate semisodium delayed release (DR) and then transitioned to valproate semisodium extendedrelease (ER). Citrome and colleagues (2004) reported on the safety, efficacy and tolerability of switching from valproate semisodium DR formulation to once-daily dosing with valproate semisodium ER in 30 individuals with schizophrenia. Patients were converted from valproate semisodium DR to ER formulation on a 1.0:1.0 basis (rounded up to the nearest 500-mg increment) for initial plasma valproate levels $\geq 85 \mu \mathrm{g} / \mathrm{mL}$ and on a 1.0: 1.2 basis for initial plasma levels of $<85 \mathrm{~g} / \mathrm{mL}$ (Citrome et al., 2004). Baseline and endpoint changes on psychopathology and adverse event ratings scales (Positive and Negative Syndrome (PANSS) and Udvalg for Klinishke Undersogelser Side Effect Rating Scale (UKU)) were minimally changed and baseline and end-point serum trough valproate semisodium levels were 80.1 and 73.1 respectively, suggesting that switch-over was well tolerated and that the differences in serum levels were of small magnitude.

Consistent with the procedures of the small trial reported here, it appears that individuals receiving valproate semisodium DR can be readily converted to valproate semisodium ER on a 1:1, or perhaps a 1.0:1.2 basis. In the case of older adults with schizophrenia, the once-daily dosing formulation was more easily remembered and accommodated into an individual's life-style than multiple-dosed valproate semisodium.

The findings of this study must be interpreted cautiously given the limitations of small sample size, open-label, add-on design and lack of a control or comparator group. Additionally, the sample mean age of approximately 61 years is not representative of the 'old-old' populations seen in some geriatric-focused studies, and results cannot necessarily be extrapolated to the oldest geriatric populations with schizophrenia.

In conclusion, this uncontrolled prospective study of adjunctive valproate semisodium in older adults with schizophrenia suggests that the addition of valproate semisodium to antipyschotic treatment may be associated with improvement in symptoms. Larger, controlled studies are needed to further evaluate the effects of adjunctive valproate semisodium on symptoms of schizophrenia in older adult populations.

\section{ACKNOWLEDGEMENTS}

This study was supported by Abbott Laboratories Dr Sajatovic has received research funding support from Abbott Laboratories and Bristol Myers Squibb, is a consultant to AstraZeneca and Glaxo Smith Kline, and is on the Speaker's Bureau for AstraZeneca. The other authors have nothing to disclose.

\section{REFERENCES}

Anderson GD. 1998. A mechanistic approach to antiepileptic drug interactions. Ann Pharmacother 32: 554-563.

Barnes TRE. 1989. A rating scale for drug-induced akathisia. $\mathrm{Br} \mathrm{J}$ Psychiatry 154: 672-676.

Basan A, Kissling W, Leucht S. 2004. Valproate as an adjunct to antipsychotics for schizophrenia: a systematic review of randomized trials. Schizophr Res 70(1): 33-37.

Calvo R, Carlos R, Erill S. 1986. Differential effects of valproic acid on the serum protein binding of lorazepam and diazepam. Int $J$ Clin Pharmacol Res 6(3): 213-215.

Casey DE, Daniel DG, Wassef AA, et al. 2003. Effect of divalproex combined with olanzapine or risperidone in patients with an acute 
exacerbation of schizophrenia. Neuropsychopharmacology $\mathbf{2 8}(1)$ : 182-192.

Centers for Disease Control (CDC). 2003. Trends in aging-United States and Worldwide. MMWR Morbidity and Mortality Weekly Report 52(6): 101-104; 106.

Citrome L, Casey DE, Wozniak P, et al. 2004. Adjunctive divalproex and hostility among patients with schizophrenia receiving olanzapine or risperidone. Psychiatr Serv 55(3): 290-294.

Citrome L, Josiassen R, Bark N, et al. 2005. Pharmacokinetics of aripiprazole and concomitant lithium and valproate. J Clin Pharmacol 45(1): 89-93.

Citrome L, Levine J, Allingham B. 2000. Changes in use of valproate and other mood stabilizers for patients with schizophrenia from 1994-1998. Psychiatr Serv 51(5): 634-638.

Citrome L, Tremeau F, Wynn PS, et al. 2004. A study of the safety, efficacy, and tolerability of switching form the standard delayed release preparation of divalproex sodium to the extended release formulation in patients with schizophrenia. J Clin Psychopharmacol 24(3): 255-259.

Folstein MF, Folstein SE, McHugh PR. 1975. 'Mini-Mental State'. A practical method for grading the cognitive state of patents for the clinician. J Psychiatr Res 12(3): 189-198.

Fuller M, Sajatovic M. 2007. Drug Information Handbook of Psychiatry, 6th edn. Lexi-Comp: Hudson, $\mathrm{OH}$.

Gobbi G, Gaudreau PO, LeBlanc N. 2006. Efficacy of topiramate, valproate, and their combination on aggression/agitation behavior in patients with psychosis. J Clin Psychopharmacol 26(5): $467-473$.

Ichikawa J, Chung YC, Dai J, Meltzer HY. 2005. Valproic acid potentiates both typical and atypical antipsychotic-induced prefrontal cortex dopamine release. Brain Res 1052(1): 56-62.

Jeste DV, Alexopoulos S, Bartels S, et al. 1999. Consensus statement on the upcoming crisis in geriatric mental health. Arch Gen Psychiatry 56(9): 848-853.

Kay SR, Fiszbein A, Opler LA. 1987. The positive and negative syndrome scale (PANSS) for schizophrenia. Schizophr Bull 13(2): 261-276.

Jones PB, Barnes TRE, Davies L, et al. 2006. Randomized controlled trial of the effect on quality of life of second vs. first-generation antipsychotic drugs in schizophrenia. Arch Gen Psychiatry 63(10): 1079-1087.

Lieberman JA, Stroup TS, McEvoy JP, et al. 2005. Effectiveness of antipsychotic drugs in patients with chronic schizophrenia. N Engl J Med 353(12): 1209-1223.

Porsteinsson AP, Tariot PN, Erb R, et al. 2001. Placebo-controlled study of divalproex sodium for agitation in dementia. Am $J$ Geriatr Psychiatry 9(1): 58-66.
Sajatovic M, Friedman SJ, Sabharwal J, Bingham CR. 2004. Clinical characteristics and length of hospital stay among older adults with bipolar disorder, schizophrenia or schizoaffective disorder, depression and dementia. Int $J$ Psychiatry Neurol 17(1): 3-8.

Sheehan DV, Lecubier Y, Sheehan KH, et al. 1998. Development and validation of a structured diagnositic psychiatric interview for DSM-IV and ICD-10. J Clin Psychiatry 59C(Suppl. 20): 22-23.

Simpson GM, Angus JWS. 1970. A rating scale for extrapyramidal side effects. Acta Psychiatr Scand Suppl 212: 11-19.

Sink KM, Holden KF, Yaffe K. 2005. Macological treatment of neuropyschiatric symptoms of dementia: a review of the evidence. JAMA 293(5): 596-608

Sival RC, Haffmans PM, Jansen PA, et al. 2002. Sodium valproate in the treatment of aggressive behavior in paitents with dementia - a randomized placebo controlled clinical trial. Int J Geriatr Psychiatry 17(96): 579-585.

Stahl SM. 2004. Anticonvulsants as mood stabilizers and adjuncts to antipsychotics: valproate, lamotrigine, carbamazepine, and oxcarbazepine and actions at voltage-gated sodium channels. J Clin Psychiatry 65(6): 738-739.

Tariot PN, Raman R, Jakimovich L, et al. 2005. Divalproex sodium in nursing home residents with possible or probably Alzheimer disease complicated by agitation. Am J Geriatr Psychiatry 13(11): 942-949.

Townsend MH, Wilson MS. 2005. Comorbid anxiety disorders and divalproex sodium use among partial hospital patients with psychotic disorders. Compr Psychiatry 46(5): 368-370.

US Department of Health, Education and Welfare. ECDEU Assessment Manual for Psychopharmacology, Revised edn, Guy W. 1976. Publication ADM 76-338.US Department of Health, Education and Welfare: Washington, DC.

Ware JE, Sherbourne CD. 1992. The MOS 36-item short-Form Health Survey (SF-36). I. Conceptual Framework and Item Selection. Med Care 30(6): 473-483.

Wassef A, Baker J, Kochan LD. 2003. GABA and schizophrenia; a review of basic science and clinical studies. J Clin Psychopharmacol 23(96): 601-640.

Wassef AA, Dott SG, Harris A, et al. 1999. Critical review of GABA-ergic drugs in the treatment of schizophrenia. $J$ Clin Psychopharmacol 19(3): 222-232.

Wassef AA, Dott SG, Harris A, et al. 2000. Randomized, placebo-controlled pilot study of divalproex sodium in the treatment of acute exacerbations of chronic schizophrenia. J Clin Psychopharmacol 20(3): 357-361.

Yesavage JA, Brink TL, Rose TL, et al. 1982. Development and Validation of a Geriatric Depression screening Scale: A Preliminary Report. J Psychiatr Res 17(1): 37-49. 